\title{
Could BCG be used to protect against COVID-19?
}

\section{Gil Redelman-Sidi[}

Nearly 100 years since it was first used in humans as a vaccine for tuberculosis, Bacillus CalmetteGuérin (BCG) has been suggested as a possible agent to prevent coronavirus disease 2019 (COVID-19). A number of studies are underway to investigate this possibility but - even if they prove effective - many questions will remain.

Studies are in progress to determine whether BCG vaccination could protect against COVID-19 infection

Division of Infectious Diseases, Memorial Sloan Kettering Cancer Center, New York, NY, USA.
The ongoing coronavirus disease 2019 (COVID-19) pandemic has renewed academic and clinical interest in an old vaccine, Bacillus Calmette-Guérin (BCG). BCG, an attenuated strain of Mycobacterium bovis, was originally developed by Albert Calmette and Camille Guérin at the start of the 20th century as a vaccine against tuberculosis. First used in humans in 1921, BCG is now one of the most widely used vaccines in infants and neonates, in whom its main utility is in the prevention of tuberculous meningitis and disseminated tuberculosis ${ }^{1}$. Importantly, BCG is also used as adjuvant immunotherapy for patients with non-muscle-invasive bladder cancer ${ }^{2}$.

In addition to its expected effect on prevention of severe disease caused by tuberculosis, BCG vaccination of children has been shown to have a number of heterologous protective effects. Most notably, BCG vaccination of neonates might decrease overall childhood mortality, including mortality unrelated to tuberculosis ${ }^{3}$, which is mainly driven by a decrease in sepsis and respiratory infections in childhood ${ }^{4}$.

Several mechanisms by which BCG provides nonspecific protection against respiratory infections have been a subject of active investigation. First, molecular similarity between BCG antigens and viral antigens could lead, after BCG vaccination, to a population of memory $\mathrm{B}$ and $\mathrm{T}$ cells that recognize both BCG and respiratory pathogens. However, this mechanism is unlikely to explain the diverse protection resulting from BCG vaccination. Second, BCG could lead to antigen-independent activation of bystander $B$ and $T$ cells, a mechanism that has been termed heterologous immunity. Finally, BCG could lead to long-term activation and reprogramming of innate immune cells. This last mechanism, which has been the subject of much interest in the past decade, has been called trained immunity ${ }^{5}$.

Trained immunity occurs through epigenetic reprogramming of monocytes at the site of infection or immunization. These monocytes undergo histone modification at promoter sites of genes encoding inflammatory cytokines, leading to long-term changes in their ability to respond to novel stimuli and resulting in an increasingly active immune response when they are reactivated. Evidence is available to support the occurrence of trained immunity in the context of BCG immunization: monocytes from adults who receive BCG vaccination exhibit increased expression of various surface markers related to activation and produce higher quantities of cytokines, such as IL-1 $\beta$, IL-6, IFN $\gamma$ and TNF, in response to infection with various pathogens compared with monocytes from adults who do not receive BCG vaccination. This protection encompasses a wide range of organisms, including bacteria such as Staphylococcus aureus, fungi such as Candida albicans and viruses such as the yellow fever virus ${ }^{6,7}$.

Investigators are now looking to test the hypothesis that heterologous protection engendered by BCG could protect against severe disease from COVID-19. Interestingly, data from a non-peer-reviewed publication provide some support for this hypothesis. The authors of this article found that parts of the world that do not have a policy of universal BCG vaccination, such as Italy and the USA, have experienced higher mortality associated with COVID-19 than places with long-standing universal BCG vaccination policies, such as South Korea and Japan ${ }^{8}$. However, this evidence is circumstantial and multiple factors other than BCG vaccination policy could explain the discrepancies in COVID-19 mortality between different places. Several studies are ongoing to determine the effect of BCG vaccination on outcomes from COVID-19, including in two high-risk populations. First, clinical trials in Australia, the Netherlands and the USA aim to test whether BCG vaccination of health-care workers could protect them from COVID-19 (see Related links). Second, studies are in progress to test the effect of BCG vaccination on prevention of severe COVID-19 infection among older people. Finally, a study in Germany is testing whether VPM1002, a recombinant vaccine strain derived from BCG, can protect either health-care workers or older patients from COVID-19 (REF.').

If these trials do show a protective effect of BCG vaccination against COVID-19, many questions will 
remain. First, how long does the heterologous immunity engendered by BCG last after vaccination? Previous studies have shown that the effects of BCG on monocytes can last for several months; however, some of these effects, particularly the enhanced ability of trained monocytes to secrete cytokines, gradually wane afterwards ${ }^{7}$. Second, what is the optimal time to vaccinate? Studies on the effect of BCG vaccination on respiratory infections in childhood and mortality demonstrate an advantage of vaccinating early in life (before 9 months of age versus later in childhood $)^{4}$. However, whether age at vaccination will also influence the effect of BCG on COVID-19 is unknown. Finally, how will the use of BCG to prevent COVID-19 affect its use to treat bladder cancer? Will use of BCG to prevent COVID-19 exacerbate the ongoing shortage of BCG to treat bladder cancer, particularly in the USA where Merck is the sole manufacturer of BCG? And will patients with bladder cancer who underwent treatment with intravesical BCG also be protected against COVID-19?

Studies are in progress to determine whether BCG vaccination could protect against COVID-19 infection. Whatever the results of these efforts, that the BCG vaccine continues to spark scientific investigations that shed new light on our understanding of immunological mechanisms over 100 years since its development is remarkable.
1. Colditz, G. A. et al. The efficacy of bacillus Calmette-Guerin vaccination of newborns and infants in the prevention of tuberculosis: meta-analyses of the published literature. Pediatrics 96, 29-35 (1995).

2. Redelman-Sidi, G., Glickman, M. S. \& Bochner, B. H. The mechanism of action of BCG therapy for bladder cancer-a current perspective. Nat. Rev. Urol. 11, 153-162 (2014).

3. Higgins, J. P. et al. Association of BCG, DTP, and measles containing vaccines with childhood mortality: systematic review. BMJ 355 , i5170 (2016).

4. Hollm-Delgado, M. G. Stuart, E. A \& Black, R. E. Acute lower respiratory infection among Bacille Calmette-Guerin (BCG)-vaccinated children. Pediatrics 133, e73-e81 (2014).

5. Goodridge, H. S. et al. Harnessing the beneficial heterologous effects of vaccination. Nat. Rev. Immunol. 16, 392-400 (2016).

6. Arts, R. J. W. et al. BCG vaccination protects against experimental viral infection in humans through the induction of cytokines associated with trained immunity. Cell Host Microbe 23, 89-100 (2018).

7. Kleinnijenhuis, J. et al. Long-lasting effects of $B C G$ vaccination on both heterologous $\mathrm{Th}_{1 / \mathrm{T}} 17$ responses and innate trained immunity. J. Innate Immun. 6, 152-158 (2014).

8. Miller, A. et al. Correlation between universal BCG vaccination policy and reduced morbidity and mortality for COVID-19: an epidemiological study. Preprint at medRxiv https://doi.org/ 10.1101/2020.03.24.20042937 (2020).

9. de Vrieze, J. Can a century-old TB vaccine steel the immune system against the new coronavirus? Science https://www.sciencemag. org/news/2020/03/can-century-old-tb-vaccine-steel-immunesystem-against-new-coronavirus\# (2020).

\section{Competing interests}

The author declares no competing interests.

\section{RELATED LINKS}

ClinicalTrials.gov search of BCG and COVID: https://clinicaltrials.gov/ ct2/results?cond=COVID\&term=bcg\&cntry=Estate $=\varepsilon c i t y=\varepsilon d i s t=$ ESearch=Search 